\title{
New progress in elucidating the relationship between cancer therapy and cardiovascular toxicity
}

\author{
Chen Wu ${ }^{1,}$, Dawei Lin ${ }^{1, \S}$, Fei Ma ${ }^{2}$, Feng Jiang ${ }^{3}$, Yaosheng Wang ${ }^{1,4, *}$ \\ ${ }^{1}$ Department of Cardiology, Xinhua Hospital Affiliated to Shanghai Jiao Tong University School of Medicine, Shanghai, China; \\ ${ }^{2}$ Department of Oncology, Xinhua Hospital Affiliated to Shanghai Jiao Tong University School of Medicine, Shanghai, China; \\ ${ }^{3}$ Clinical Research \& Innovation Unit, Xinhua Hospital Affiliated to Shanghai Jiao Tong University School of Medicine, Chongming Branch, \\ Shanghai, China; \\ ${ }^{4}$ Clinical Research \& Innovation Unit, Xinhua Hospital Affiliated to Shanghai Jiao Tong University School of Medicine, Shanghai, China.
}

\begin{abstract}
SUMMARY Onco-cardiology is an emerging field linking cancer with cardiovascular injury. Understanding the mechanism of cardiac injury helps improve the quality of life of cancer survivors. A series of studies on adverse reactions to cancer or oncological treatments has indicated that adverse cardiovascular events related to cancer treatments may occur over a longer period of survival, and even years after therapy has concluded. Current cancer therapies, including chemotherapy, radiotherapy, targeted therapy, and immunotherapy, have been found to have latent cardiovascular toxicity. These toxic effects are often progressive and irreversible and ultimately lead to cardiovascular events such as heart failure, hypertension, coronary heart diseases, arrhythmia, and thromboembolism. Therefore, more emphasis should be placed on revealing the mechanism of cancer treatment-related cardiovascular toxicity. This would help to guide prevention, diagnosis, and treatment of CVDs in cancer survivors.
\end{abstract}

Keywords onco-cardiology, cardiovascular toxicity, cardiovascular diseases, cancer

\section{Introduction}

Cardiovascular diseases (CVDs) and cancer are the two leading causes of death worldwide. Mortality from cancer has been steadily declining over the past few decades, largely because of early detection strategies, improved surgical approaches, and advances in cancer therapeutics. However, recent data have indicated that CVDs are becoming the second leading cause of longterm morbidity and mortality among cancer survivors. This is largely due to the following reasons: $i$ ) Cancer and CVDs involve many common risks and pathologic factors, like smoking, diabetes, and aging (1-3); ii) Various cancer treatments cause direct or indirect damage to the cardiovascular system. According to previous investigations, over $60 \%$ of patients were prescribed cardiovascular risk drugs during cancer treatments $(4,5)$. Conventional chemotherapy and targeted therapies are associated with an increased risk of cardiac damage including heart failure (HF), hypertension, arrhythmia, thromboembolism, and ischemic cardiomyopathy (CM), and may be life-threatening in some instances.

Oncologists puzzled by the necessity of cardiovascular assessment and monitoring when dealing with patients with tumor. The field of onco-cardiology has developed in response to a fusion of relevant theories in cardiology and oncology to optimize care for patients with cancer. Thus, further studies on the epidemiology and pathophysiology of cardiovascular damage \& disfunction in cancer survivors need to be promptly conducted to identify screening, preventive, and therapeutic approaches.

\section{Epidemiology: Cancer treatments and CVDs}

The overall incidence of cancer increases steadily with age for over $80 \%$ of patients between the ages of 60 and 80. The International Agency for Research on Cancer (IRCA) has reported that there were approximately 19.3 million new patients with cancer worldwide and nearly 10 million deaths in 2020 (6). In China, there were close to 4.57 million new cancer cases in 2020, accounting for about a quarter of new patients with cancer worldwide. The number of patients found to have cancer is estimated to increase by $60 \%$ in 2030 .

As a result of continued advances in cancer treatments, the survival rate has increased significantly. About $75 \%$ of cancer survivors suffer from one or more chronic diseases, and the leading cause of death for these survivors is CVDs (7-9). The largest cohort analysis of 
cancer and CVDs in China reported that $18 \%$ of patients with cancer have at least one cardiovascular risk factor and that $5 \%$ of patients with cancer have CVDs (4). Patients with cancer and CVDs have a higher mortality risk than patients without CVDs. In addition, a 2008 study by Menna, Salvatorelli, \& Minotti (in Chemical Research in Toxicology) found that many decades after diagnosis and treatment, patients with cancer had a 15fold higher rate of $\mathrm{HF}$, a 9-fold higher rate of stroke, and a 10-fold higher incidence of coronary atherosclerosis $(10,11)$. Within 5 to 10 years after cancer treatments, more than $50 \%$ of survivors have subclinical signs of heart and blood vessel damage $(12,13)$.

\section{Mechanism of cancer treatment-related cardiovascular toxicity}

Direct compression or infiltration of the heart by tumor, cytokines and growth factors secreted by tumor cells, and endocrine factors released by tissues surrounding tumors degrade the normal structure and function of the heart. The control of primary disease is the best way to alleviate these effects. However, mounting evidence has suggested that cancer treatments, such as drugs, surgery, and radiation (Table 1), induce cardiovascular impairment that may be related to cardiomyocyte apoptosis, mitochondrial damage, oxidative stress, proinflammatory response, and endothelial cell damage (Figure 1).

\subsection{Cardiac toxicity}

The structure and electrical behavior of the myocardium are the two leading components that maintain the normal physiological function of the heart. Various cancer treatments disrupt normal cell, tissue, and organ function, degrading cardiac function and ultimately leading to CVDs.

\subsubsection{Structural dysfunction of the myocardium}

Cardiomyocyte apoptosis has been reported to be a key mechanism of cancer treatment-induced cardiotoxicity, and it is frequently accompanied by oxidative stress and mitochondrial injury. Some evidence suggests that anthracyclines, one of the most widely used chemotherapeutic agents, induce apoptosis of cardiomyocytes. Anthracycline exposure reduces the number of mesenchymal progenitor and circulating cells, and thus reduce cardiorepartive capacity when the heart is exposed to stress (14). In addition, anthracyclines act on topoisomerase-II, which has been found to be one of the direct target molecules of cardiotoxic drugs. Dysfunction of topoisomerase II exacerbates the breakage of DNA, mitochondrial DNA damage, and oxidative stress, it prevents ligase repair, and it up-regulates the expression of MAPK,
Table 1. Mechanism of cardiovascular toxicity induced by cancer treatments

\begin{tabular}{|c|c|c|}
\hline Mechanism & Cancer treatments & Related CVDs \\
\hline $\begin{array}{l}\text { Cardiac } \\
\text { apoptosis }\end{array}$ & $\begin{array}{l}\text { - Anthracyclines } \\
\text { - Antimetabolites } \\
\text { - PKIs } \\
\text { - Radiation } \\
\text { - Topoisomerase II inhibitors }\end{array}$ & $\begin{array}{l}\text { HF } \\
\text { Arrythmia } \\
\text { Heart valve disease }\end{array}$ \\
\hline $\begin{array}{l}\text { Endothelial } \\
\text { damage }\end{array}$ & $\begin{array}{l}\text { - Alkylating agents } \\
\text { - Anthracyclines } \\
\text { - PKIs } \\
\text { - Taxanes } \\
\text { - Proteasome inhibitors } \\
\text { - Radiation } \\
\text { - Platinum } \\
\text { - VEGF inhibitors }\end{array}$ & $\begin{array}{l}\text { CAD } \\
\text { Hypertension } \\
\text { Thromboembolism }\end{array}$ \\
\hline $\begin{array}{l}\text { Mitochondrial } \\
\text { injury }\end{array}$ & $\begin{array}{l}\text { - Anthracyclines } \\
\text { - TKIs }\end{array}$ & $\begin{array}{l}\text { HF } \\
\text { Arrythmia } \\
\text { CAD }\end{array}$ \\
\hline $\begin{array}{l}\text { Oxidative } \\
\text { stress }\end{array}$ & $\begin{array}{l}\text { - Alkylating agents } \\
\text { - Anthracyclines } \\
\text { - Antimetabolites } \\
\text { - Mitomycin C }\end{array}$ & $\begin{array}{l}\text { HF } \\
\text { Pericarditis } \\
\text { CAD }\end{array}$ \\
\hline $\begin{array}{l}\text { Proinflammatory } \\
\text { response }\end{array}$ & $\begin{array}{l}\text { - Anthracyclines } \\
\text { - Antimetabolites } \\
\text { - Interferon- } \alpha\end{array}$ & $\begin{array}{l}\text { HF } \\
\text { Pericarditis } \\
\text { Arrythmia } \\
\text { CAD }\end{array}$ \\
\hline $\begin{array}{l}\text { Cytokine release } \\
\text { syndrome }\end{array}$ & $\begin{array}{l}\text { - Alkylating agents } \\
\text { - Anthracyclines } \\
\text { - Antimetabolites } \\
\text { - Monoclonal antibodies } \\
\text { - Amsacrine } \\
\text { - Platinum } \\
\text { - Melphalan }\end{array}$ & $\begin{array}{l}\text { Thromboembolism } \\
\text { Arrythmia } \\
\text { HF } \\
\text { CAD }\end{array}$ \\
\hline $\begin{array}{l}\text { Abnormal } \mathrm{Ca}^{2+} \\
\text { homeostasis }\end{array}$ & $\begin{array}{l}\text { - Anthracyclines } \\
\text { - Arsenic trioxide } \\
\text { - Taxanes }\end{array}$ & Arrythmia \\
\hline
\end{tabular}

Abbreviation: PKIs, protein kinase inhibitors; TKIs, Tyrosine kinase inhibitors; HF, heart failure; CAD, coronary atherosclerosis; VEGF inhibitors, vascular endothelial growth factor inhibitors.

JNK, p38, and protein kinase B. Thus affecting $\mathrm{Ca}^{2+}-$ regulating proteins or $\mathrm{Ca}^{2+}$ channels and leading to events such as $\mathrm{Ca}^{2+}$ overload and cardiac remodeling (15-17). Another anti-cancer agent involved in apoptosis is imatinib, which produces a dose-dependent collapse of the mitochondrial membrane potential that damages cardiomyocyte mitochondria. At the subcellular level, a lack of functional mitochondria causes a decrease in intracellular ATP content, an endoplasmic reticulum stress response, and increased release of cytochrome $\mathrm{C}$ into the cytoplasm, thus accelerating cardiomyocyte apoptosis (18).

Another important factor causing abnormalities in the structure of the heart is due to cancer treatmentrelated cardiomyocyte and interstitial fibrosis. Human epidermal growth factor receptor 2 (HER2) inhibitor is a targeted drug to treat breast cancer that interferes with the heterodimerization of ErB2 and ErB4 in cardiomyocytes, thus eliminating the protective effect of neuregulin 1, which helps to repair cardiomyocytes 


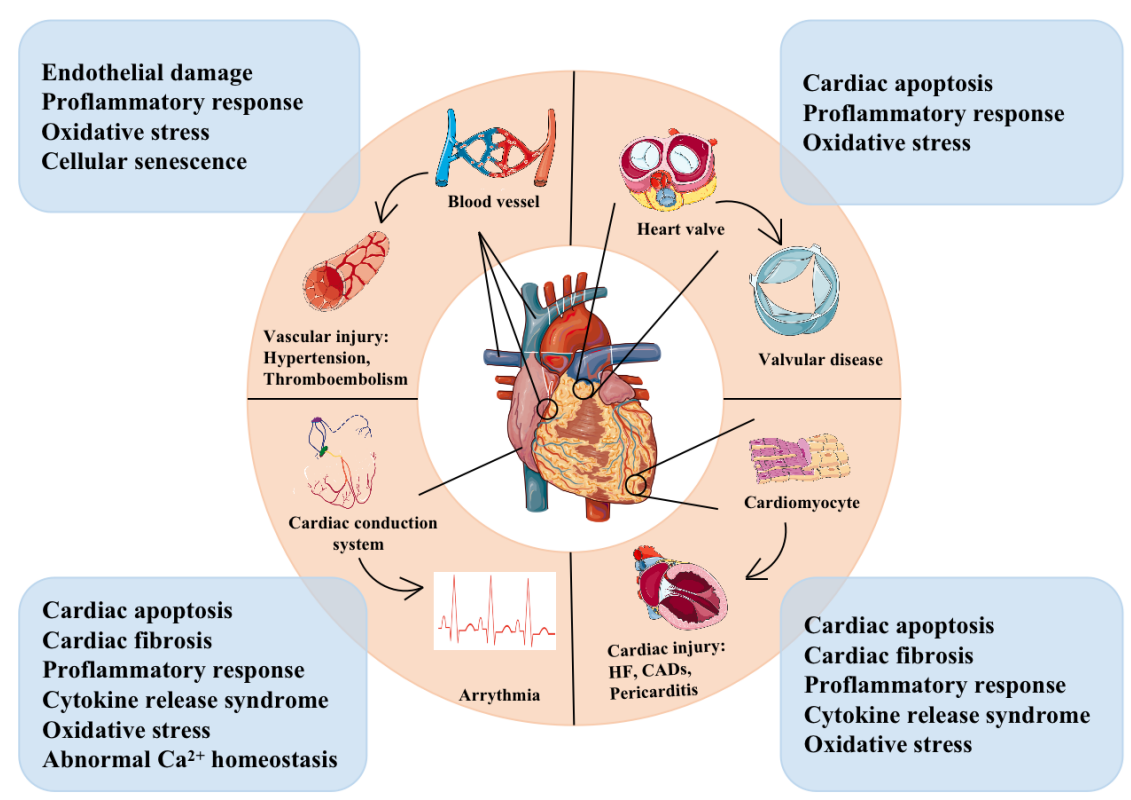

Figure 1. The mechanism of cardiovascular toxicity induced by cancer treatments.

and maintain homeostasis $(19,20)$. In addition to cardiac fibrosis directly caused by drugs, physical therapy also significantly accelerates this process. Radiation therapy continues to advance, and it has become the primary modality for clinical treatment of malignancies. Radiation therapy kills cancer cells and prevents them from returning via a variety of mechanisms. However, it simultaneously kills neighboring normal cells, so the radiation dose and volume exposed must be tightly controlled. Prolonged radiation exposure affects cardiac capillary endothelial cells, leading to their proliferation, damage, swelling, and degeneration, and it significantly reduces the number of capillaries (21). Capillary network damage is associated with decrease in the myocardial blood supply, eventually contributing to the development of myocardial fibrosis. Radiotherapy also induces fibrosis of the heart valves and cusps (22).

In addition to mechanisms mentioned thus far, proinflammatory response and cytokine release syndrome (CRS) often occurs together during immunotherapy and biological treatment. Immune effector cells crosslink with tumor cells, contributing to the release of a large number of cytokines and inflammatory factors. Cytokines are mainly derived from the target cells themselves and immune cells recruited to the tumor. Aggregation of cytokines can lead to excessive activation of immune cells such as macrophages, which create a positive feedback loop that promotes the activation of cytokines such as IL-1, IL-6, IL-8, IL-10, and MCP1 , eventually leading to a cytokine storm and excessive inflammation $(23,24)$.

\subsubsection{Myocardial electrical dysfunction}

Studies have indicated that cancer treatments regulate cardiomyocyte ion channels. HER2 inhibitors regulate cardiac sympathetic nerve tension and affect a variety of ion channels, such as $\mathrm{I}_{\mathrm{to}}, \mathrm{I}_{\mathrm{K} 1}$, and $\mathrm{I}_{\mathrm{K}, \mathrm{Ach}}$ (3). Arsenic trioxide, which is often used to treat hematologic cancers, blocks $\mathrm{I}_{\mathrm{Kr}}$ and $\mathrm{I}_{\mathrm{Ks}}$ and activates $\mathrm{I}_{\mathrm{K}, \text { Ach }}(25)$. An increase in myocyte automaticity and altered expression of ion channels induces prolongation of the QT interval and early post-depolarization and it triggers abnormal cardiac electrical activity.

In addition, calcium homeostasis plays a key role in myocardial excitation-contraction coupling, which is involved in the changes in cardiac electrical activity. Studies have indicated that paclitaxel increases the expression of neuronal calcium sensor 1 (NCS-1), it modulates the release of calcium from cells depending on the inositol-1,4,5-triphosphate receptor (InsP3R), and it affects calcium signaling, calcium oscillation, and calcium homeostasis in cardiomyocytes. Thus disrupting the normal electrophysiology of the myocardium (26).

\subsection{Vascular toxicity}

Cardiotoxicity has received extensive scholarly attention, but vascular toxicity cannot be ignored. Endothelial injury is a key feature of vascular toxicity and it is caused by cell aging, oxidative stress, and pro-inflammatory response.

Cisplatin and carboplatin are platinum-based agents that have been in widespread use for many years to treat several forms of cancer, and platinum-based agents are considered to be vasotoxic. Cisplatin can still be detected in serum several years after treatment, and it continues to affect vascular endothelium (27). Cisplatin exposure activates endothelial cells and then up-regulates intracellular adhesion molecule 1, tissue- 
type plasminogen activator, and plasminogen activator inhibitor type 1 in endothelial cells, suggesting an acceleration of atherosclerosis $(11,27)$. After many years of cisplatin therapy, patients have an increased carotid intima media thickness (C-IMT) and aortic IMT (A-IMT). Many other types of anticancer drugs appear to be vasotoxic. 5-Fu and capecitabine are commonly used antimetabolites that promote oxidative stress, causing cellular damage and readily inducing endothelial injury followed by thrombosis (28).

Radiotherapy is known to cause the shortening and dysfunction of telomeres, thus accelerating cellular senescence. Senescence of vascular endothelial cells increases the production of reactive oxide species (ROS) and decreases the vascular protective effect of nitric oxide, contributing to the senescence-induced apoptosis of endothelial cells. Cellular senescence is thought to be a gradual decline in the ability of cells to cope with various stresses that may trigger chronic pathological conditions, including CVDs (29).

\section{CVDs associated with cancer treatments}

Therapy of a longer duration is considered to involve an increased risk of cardiovascular events such as HF, hypertension, CAD, arrhythmia, thromboembolism, heart valve disease, and pericarditis. These events are common, and they involve a high rate of morbidity and disability. Therefore, the cardiovascular system should be monitored, and cardiovascular events prevented in all patients with cancer.

\subsection{HF}

HF is one of the most common complications in patients with cancer, and it influences the overall outcomes for those patients, particularly the elderly. Conventional chemotherapeutics, such as anthracyclines, antimetabolites, and cyclophosphamide, can induce permanent myocardial cell injury, leading to left ventricular (LV) dysfunction (LVD) and diastolic and systolic dysfunction and eventually progressing to acute or chronic HF (30). Anthracyclines, especially doxorubicin, are the greatest concern, since they can lead to HF and significantly shortener lifespan. Anthracyclinerelated LVD has historically been considered to be dosedependent, cumulative, and progressive; over $50 \%$ of patients receiving anthracycline-based chemotherapy have a certain degree of cardiac dysfunction 10-20 years after chemotherapy, 5\% have obvious HF, and the mortality rate is close to $60 \%(31)$. A study has indicated that myocyte injury may also be reversible if LVD, diastolic as well as systolic dysfunction are detected early, and appropriate HF-based treatment is instituted (32).

Surveillance strategies are currently based on expert consensuses. An integrated approach combining cardiac biomarkers as well as imaging data plays a major role in identification, assessment, and monitoring of antitumor drug-induced cardiotoxicity. Troponins may facilitate the detection of cardiotoxicity in the preclinical phase and help to predict the severity of future LVD in patients treated with antitumor drugs $(33,34)$.

The left ventricular ejection fraction (LVEF) is the most commonly used parameter of cardiac function that independently predicts short-term and long-term mortality from cardiovascular events. The Clinical Practice Guidelines of the American Society of Clinical Oncology (ASCO) recommend assessment of LVEF prior to and during exposure to known cardiotoxic agents in high-risk patients (35). Recent improvements in imaging technology have led to more sensitive and precise assessment of cardiac function. New techniques, including spot tracking and measurement of strain, make up for the technical shortcomings of conventional ultrasound (36). These imaging technologies have enabled early detection of structural injury to the heart and cardiac injury as a result of cancer therapy.

The primary goal of treating cancer treatment-related HF is maintaining the LVEF and limiting myocardial remodeling. There is growing evidence suggesting that several agents, such as $\beta$-blockers, angiotensin antagonists, statins, angiotensin-converting enzyme inhibitors (ACEIs), and angiotensin receptor blockers (ARBs), play a cardioprotective role in preventing cancer treatment-induced cardiotoxicity. However, patients receiving anthracycline-based chemotherapy have a significant increase in adverse reactions to ACEIs/ARBs, such as a dry cough, hypotension, hyperkalemia, and angioedema (37). For these patients, carvedilol is a better choice to reduce $\mathrm{HF}$.

\subsection{Hypertension}

Hypertension is a common comorbidity in patients with cancer. Nearly $70 \%$ of patients with prostate or bladder cancer develop hypertension. Moreover, the toxicity of anticancer drugs accelerates the development of hypertension. Monoclonal antibodies (such as bevacizumab), TKIs, and vascular endothelial growth factor (VEGF) signaling pathway (VSP) inhibitors disrupt endothelial cell receptor signaling, which affects the activity of nitric oxide synthase, nitric oxide levels, and endothelin synthesis. Increased vasoconstriction capacity and decreased peripheral angiogenesis induce hypertension. In addition, alkylating agents, taxanes, platinum-based agents, and neuroendocrine drugs can also cause hypertension.

The primary goal of treating patients with hypertension is to minimize damage to target organs including the brain, kidneys, and heart by standard management of blood pressure (BP). Effectively lowering $\mathrm{BP}$ reduces morbidity and mortality. The European Society of Cardiology recommends a target systolic $\mathrm{BP}(\mathrm{SBP})<140 \mathrm{mmHg}$ and a diastolic $\mathrm{BP}(\mathrm{DBP})<90$ 
mmHg for patients taking a VSP inhibitor (7). BP should be actively monitored throughout treatment, with weekly assessments during the first cycle of treatment and assessments at least every 2 to 3 weeks during the remaining cycles. In addition, the target BP should be adjusted to $<130 / 80 \mathrm{mmHg}$ for patients with multiple pre-existing risk factors for adverse consequences of high BP, such as chronic kidney disease (38).

Specific classes of antihypertensives have not been recommended for patients with cancer treatmentrelated hypertension. ACEIs or ARBs, calcium channel blockers (CCBs), and $\beta$-blockers are the treatment of choice. However, non-dihydropyridine calcium channel blockers (non-DHP CCBs), such as verapamil and diltiazem, should be avoided in patients receiving sunitinib or sorafenib due to underlying drug interactions (7). Multi-agent antihypertensive therapy is the preferred treatment for patients with cancer and poorly controlled BP. However, clinical guidelines for the management of hypertension recommend the temporary withdrawal of anticancer drugs if BP cannot be controlled and hypertension-related adverse events occur (39).

\subsection{Coronary artery diseases (CADs)}

The coexistence of CAD and cancer is largely driven by common risk factors (such as aging, smoking, obesity, and diabetes) and the increasing use of cancer treatments. $\mathrm{CAD}$ is triggered by anti-cancer reagents in cancer patients due to increased low-density lipoprotein levels, platelet activation, endothelial damage, and vasospasms (40). The combination of bevacizumab, bleomycin, and vinblastine increases the long-term risk of CAD and myocardial infarction by 1.5 - to 7-fold (41). Moreover, radiotherapy has also been found to have negative effects: coronary events occur within 5 years after initial exposure to radiation, lasts up to 30 years postexposure, and they increase linearly with an increase in the radiation dose (42).

As is true for all patients with a heart condition, non-invasive stress testing, ECG, CTA, and coronary angiography represent important modalities for the assessment and management of cancer survivors with $\mathrm{CAD}(43,44)$. ECG and cardiac biomarkers are recommended for initial diagnosis of symptoms suggestive of an acute coronary syndrome (ACS). Clinical practice guidelines recommend the temporary withdrawal of chemotherapy if ECG from patients receiving pyrimidine analogue indicates acute myocardial ischemia, such as ST segment changes or T wave inversion.

In patients with angina that fails to respond to optimal medical therapy or in patients with a high TIMI score, invasive evaluation and treatment should be considered. Percutaneous coronary intervention (PCI) is the gold standard for ST-segment elevation myocardial infarction (STEMI), non-ST-segment elevation myocardial infarction (NSTEMI)/unstable angina (40). After surgery, patients receive routine anticoagulation therapy for at least 12 months. For patients with cancer and a high risk of bleeding, the European Society of Cardiology (ESC) recommends triple therapy [dual anti-platelet therapy (DAPT) and an oral anticoagulant (OAC)] for one month. Patients should subsequently receive antiplatelet drug and OAC for 11 months. If the risk of bleeding is extremely high, administration of an antiplatelet drug and an OAC for 12 months should be considered, and then OAC can be taken alone (45).

Other important recommendations for patients with cancer and CAD are control of symptoms, prevention of the progression of atherosclerosis, and prevention of the development of acute coronary syndrome via active medical treatment, and particularly with statins and $\beta$-blockers.

\subsection{Arrhythmia}

Kravchenko et al. reported that $28.6 \%$ of patients with lung cancer had arrythmia, including those who received chemotherapy or radiotherapy (46). In general, cancer therapy-related arrhythmias can be differentiated into bradycardia and tachycardia such as atrial fibrillation (AF), ventricular tachycardia (VT), long Q-T syndrome, and heart block.

Cardiac arrhythmias were first found to occur with paclitaxel, which mainly induces episodes of asymptomatic bradycardia. All other rare paclitaxelinduced arrythmias, including supraventricular tachycardia and premature ventricular, are selflimiting and resolve $48 \mathrm{~h}$ after treatment is stopped. Radiation therapy is another classic cancer treatment that produce different forms of bradycardia, up to a grade III atrioventricular block. Exposure to radiation causes fibrosis in the conduction system, including the sinus node, atrioventricular and His bundle, and bundle branches.

QTc prolongation and related VT has been noted with arsenic trioxide and various TKIs. These effects are attributed to an increase in the inward current and a decrease in the outward current in the ventricle.

$\mathrm{AF}$ is the most frequent sustained arrhythmia in clinical practice, and its prevalence increases with age. Initially, reported risk factors for AF were neoplastic infiltration, mechanical pressure on the heart, or a complication of oncological thoracic surgery. The association between cancer therapy and AF was subsequently identified. During prolonged treatment, the release of proinflammatory cytokines, abnormalities in calcium homeostasis, direct myocardial damage, and an increase in vagal and adrenergic tones can interfere with the electrical conduction system of the heart and serve as major risk factors for $\mathrm{AF}$.

ECG is gold standard for diagnosis of arrhythmia. Clinicians should investigate the QT interval with a 
routine 12-lead ECG and calculate the QTc (heart ratecorrected QT interval) both before and after cancer treatments (47). During treatment, discontinuation or adjustment of the anti-cancer regimen should be considered for patients with a QTc $>500 \mathrm{~ms}$, QTc prolonged more than $60 \mathrm{~ms}$, or new arrhythmia. In addition, 24-hour continuous Holter recording is helpful in diagnosing suspected arrhythmias, and establishing their frequency, relating them to symptoms, and assessing the response to therapy (48).

Management and prevention of arrhythmia in oncology patients have the same goals as in the general population. Amiodarone and $\beta$-blockers are used as first-line treatments. $\mathrm{Ca}^{2+}$ channel blockers (CCBs) are recommended to reduce the heart rate, but recent studies have indicated that ibrutinib increases the plasma levels of CCBs, amiodarone, and digoxin $(47,48)$. Therefore, caution is recommended when these drugs are combined to manage tachyarrhythmia.

Adequate anticoagulation therapy is the cornerstone of AF treatment, but it must be carefully administered to patients receiving cancer therapies. CHADS2 (CHA2DS2-VASc) and HAS-BLED scoring standards are currently the general methods for assessing AF. However, neither consider cancer and cancer treatmentinduced hypercoagulability. Thus, both the score and the patient's condition need to be considered when determining whether to administer anticoagulant therapy.

\subsection{Thromboembolism}

Hypercoagulability associated with cancer seems to be a major problem leading to thrombosis. Thromboembolism can be divided into venous thrombosis and arterial thrombosis. Venous thrombosis often occurs in patients with advanced cancer; it poses a huge burden and has a poor prognosis (49). Arterial embolism is mainly evident as acute coronary syndrome, acute ischemic stroke, or peripheral vascular disease. Coronary thrombosis is the most common form of acute coronary syndrome. Cancer treatments accelerate thrombosis due to injury to the vascular endothelium and disruption of normal coagulation (50). Anti-angiogenic drugs or TKIs lead to arterial thrombosis such as thrombosis or embolism; thalidomide or lenalidomide are major causes of venous thrombosis, which manifests as deep vein or pulmonary embolism.

Excellent tumor control is associated with a decreased risk of thromboembolism. Therefore, anti-cancer drugs are not considered to be absolutely contraindicated. Given a high risk of arterial embolism, VEGF should be avoided and aspirin or clopidogrel should be used to prevent thromboembolism. Warfarin and low molecular weight heparin $(\mathrm{LMWH})$ are recommended to treat patients with an increased risk of venous thrombosis (51). A point worth mentioning is that deep vein thrombosis is a serious complication after surgery for cancer, regardless of the type of cancer or the patient's age or sex. Khorana scoring can be used to predict the risk of chemotherapyassociated VET. In high-risk patients undergoing surgery, bleeding and the risk of an embolism should be assessed preoperative and anticoagulants should be administered to prevent embolism.

\subsection{Other CVDs}

Pericarditis, myocarditis, and heart valve disease are seen in patients with cancer, and especially those undergoing radiotherapy (21). Acute radiation pericarditis typically occurs within days to a few weeks after treatment and gradually develops into chronic pericarditis, which manifests as pericardial effusion or constrictive pericarditis. Mitral valve dysfunction is the main feature of radiation-related heart valve disease, and the degree of damage is correlated with the radiation dose.

The main methods of monitoring pericarditis, myocarditis, and heart valve disease include ECG and echocardiography. Cardiac ultrasound is generally the method of choice for monitoring pericarditis and heart valve disease, and it can be used to evaluate the structure and function of the heart valves and pericardium (34). A conventional 12-lead ECG is recommended as an initial diagnostic test for patients suspected of having myocarditis.

Clinical data on the prevention and treatment of cancer treatment-related pericardial and valvular disease are limited. As in patients who do not have cancer, conventional surgical resection and interventional valve replacement are indicated for patients with cancer treatment-related pericardial or valvular disease. Due to the potential adverse effects of radiotherapy, a lower radiation dose or new techniques to precisely locate and expose less of the heart are crucial to reducing valvular and pericardial injury.

\section{Conclusion}

Improvements in cancer diagnosis and treatment have led to a significantly increased survival rate for patients with cancer. Long-term quality of life after therapy has become a topic of increasing interest for both cancer survivors and experts. Cancer therapies cause shortterm and long-term adverse reactions involving the heart and circulation and they exacerbate existing CVDs. Therefore, large-scale prospective studies need to be conducted and the mechanism of cancer treatmentrelated cardiovascular toxicity needs to be understood further to improve guidelines on appropriate monitoring, prevention, and treatment of cancer treatment-related cardiovascular events.

\section{Acknowledgements}

The authors wish to thank all of the investigators and 
supporters involved in this study.

Funding: This work was supported by grants from the National Natural Science Foundation of China (grant no. 81974022) and the Chongming Branch of Xinhua Hospital Affiliated with the School of Medicine, Shanghai Jiao Tong University.

Conflict of Interest: The authors have no conflicts of interest to disclose.

\section{References}

1. Bibbins-Domingo K, Force USPST. Aspirin Use for the Primary Prevention of Cardiovascular Disease and Colorectal Cancer: U.S. Preventive Services Task Force Recommendation Statement. Ann Intern Med. 2016; 164:836-845.

2. Koene RJ, Prizment AE, Blaes A, Konety SH. Shared Risk Factors in Cardiovascular Disease and Cancer. Circulation. 2016; 133:1104-1114.

3. Ridker PM, MacFadyen JG, Thuren T, Everett BM, Libby P, Glynn RJ; CANTOS Trial Group. Effect of interleukin$1 \beta$ inhibition with canakinumab on incident lung cancer in patients with atherosclerosis: exploratory results from a randomised, double-blind, placebo-controlled trial. The Lancet. 2017; 390:1833-1842.

4. Dong Liu, Zhiqiang Ma, Jingang Yang, Min Zhao, Huiping Ao, Xiaodong Zheng, Qianfa Wen, Yuejin Yang, Jiangyun You, Shubin Qiao, Yuan J. Prevalence and prognosis significance of cardiovascular disease in cancer patients: a population-based study. Aging (Albany NY). 2019; 11:7948-7960.

5. Handy CE, Quispe R, Pinto X, Blaha MJ, Blumenthal RS, Michos ED, Lima JAC, Guallar E, Ryu S, Cho J, Kaye JA, Comin-Colet J, Corbella X, Cainzos-Achirica M. Synergistic Opportunities in the Interplay Between Cancer Screening and Cardiovascular Disease Risk Assessment: Together We Are Stronger. Circulation. 2018; 138:727-734.

6. Cree IA, Indave Ruiz BI, Zavadil J, et al. The International Collaboration for Cancer Classification and Research. Int J Cancer. 2021; 148:560-571.

7. Kidoguchi S, Sugano N, Tokudome G, Yokoo T, Yano Y, Hatake K, Nishiyama A. New Concept of OncoHypertension and Future Perspectives. Hypertension. 2021; 77:16-27.

8. Okura Y, Takayama T, Ozaki K, Tanaka H, Seki H, Takenouchi T, Sato N, Minamino T. Burden of cardiovascular disease in Japanese cancer patients and survivors: a single cancer-center study in Niigata City. Int J Clin Oncol. 2019; 24:196-210.

9. Okura Y, Ozaki K, Tanaka H, Takenouchi T, Sato N, Minamino T. The Impending Epidemic of Cardiovascular Diseases in Patients With Cancer in Japan. Circ J. 2019; 83:2191-2202.

10. Cardinale D, Bacchiani G, Beggiato M, Colombo A, Cipolla CM. Strategies to prevent and treat cardiovascular risk in cancer patients. Semin Oncol. 2013; 40:186-198.

11. Lenneman CG, Sawyer DB. Cardio-Oncology: An Update on Cardiotoxicity of Cancer-Related Treatment. Circ Res. 2016; 118:1008-1020.

12. Melissa M Hudson, Kirsten K Ness, James G Gurney, Daniel A Mulrooney, Wassim Chemaitilly, Kevin R Krull,
Daniel M Green, Gregory T Armstrong, Kerri A Nottage, Kendra E Jones, Charles A Sklar, Deo Kumar Srivastava , Robison LL. Clinical ascertainment of health outcomes among adults treated for childhood cancer. JAMA. 2013; 309:2371-2381.

13. Akam-Venkata J, Franco VI, Lipshultz SE. Late Cardiotoxicity: Issues for Childhood Cancer Survivors. Curr Treat Options Cardiovasc Med. 2016; 18:47.

14. Huang C, Zhang X, Ramil JM, Rikka S, Kim L, Lee Y, Gude NA, Thistlethwaite PA, Sussman MA, Gottlieb RA, Gustafsson AB. Juvenile exposure to anthracyclines impairs cardiac progenitor cell function and vascularization resulting in greater susceptibility to stress-induced myocardial injury in adult mice. Circulation. 2010; 121:675-683.

15. Narezkina A, Nasim K. Anthracycline Cardiotoxicity. Circ Heart Fail. 2019; 12:e005910.

16. Songbo M, Lang H, Xinyong C, Bin X, Ping Z, Liang S. Oxidative stress injury in doxorubicin-induced cardiotoxicity. Toxicol Lett. 2019; 307:41-48.

17. Osataphan N, Phrommintikul A, Chattipakorn SC, Chattipakorn N. Effects of doxorubicin-induced cardiotoxicity on cardiac mitochondrial dynamics and mitochondrial function: Insights for future interventions. J Cell Mol Med. 2020; 24:6534-6557.

18. Singh AP, Umbarkar P, Tousif S, Lal H. Cardiotoxicity of the BCR-ABL1 tyrosine kinase inhibitors: Emphasis on ponatinib. Int J Cardiol. 2020; 316:214-221.

19. Calvillo-Arguelles O, Abdel-Qadir H, Suntheralingam S, Michalowska M, Amir E, Thavendiranathan P. Trastuzumab-Related Cardiotoxicity and Cardiac Care in Patients With HER2 Positive Metastatic Breast Cancer. Am J Cardiol. 2020; 125:1270-1275.

20. Conduit C, de Boer RH, Lok S, Gibbs P, Malik L, Loh Z, Yeo B, Greenberg S, Devitt B, Lombard J, Nottage M, Collins I, Torres J, Nolan M, Nott L. Real-world impact of anti-HER2 therapy-related cardiotoxicity in patients with advanced HER2-positive breast cancer. Asia Pac J Clin Oncol. 2020; 16:356-362.

21. Wang H, Wei J, Zheng Q, Meng L, Xin Y, Yin X, Jiang X. Radiation-induced heart disease: a review of classification, mechanism and prevention. Int J Biol Sci. 2019; 15:21282138.

22. Sioka C, Fotopoulos A. Cardiotoxicity of thoracic radiotherapy. Radiother Oncol. 2020; 151:287.

23. Kotch C, Barrett D, Teachey DT. Tocilizumab for the treatment of chimeric antigen receptor $\mathrm{T}$ cell-induced cytokine release syndrome. Expert Rev Clin Immunol. 2019; 15:813-822.

24. Godel P, Shimabukuro-Vornhagen A, von BergweltBaildon M. Understanding cytokine release syndrome. Intensive Care Med. 2018; 44:371-373.

25. Tamargo J, Caballero R, Delpon E. Cancer chemotherapy and cardiac arrhythmias: a review. Drug Saf. 2015; 38:129-152.

26. Zhang K, Heidrich FM, DeGray B, Boehmerle W, Ehrlich BE. Paclitaxel accelerates spontaneous calcium oscillations in cardiomyocytes by interacting with NCS-1 and the InsP3R. J Mol Cell Cardiol. 2010; 49:829-835.

27. Stelwagen J, Lubberts S, Steggink LC, et al. Vascular aging in long-term survivors of testicular cancer more than 20 years after treatment with cisplatin-based chemotherapy. Br J Cancer. 2020; 123:1599-1607.

28. Otto C, Hahlbrock T, Eich K, Karaaslan F, Jurgens C, Germer CT, Wiegering A, Kammerer U. Antiproliferative 
and antimetabolic effects behind the anticancer property of fermented wheat germ extract. BMC Complement Altern Med. 2016; 16:160.

29. Ping Z, Peng Y, Lang H, Xinyong C, Zhiyi Z, Xiaocheng W, Hong Z, Liang S. Oxidative Stress in RadiationInduced Cardiotoxicity. Oxid Med Cell Longev. 2020; 2020:3579143.

30. Curigliano G, Cardinale D, Dent S, Criscitiello C, Aseyev O, Lenihan D, Cipolla CM. Cardiotoxicity of anticancer treatments: Epidemiology, detection, and management. CA Cancer J Clin. 2016; 66:309-325.

31. L J Steinherz, P G Steinherz, C T Tan, G Heller, Murphy ML. Cardiac toxicity 4 to 20 years after completing anthracycline therapy. JAMA. 1991; 266:1672-1677.

32. Cardinale D, Colombo A, Lamantia G, Colombo N, Civelli M, De Giacomi G, Rubino M, Veglia F, Fiorentini C, Cipolla CM. Anthracycline-induced cardiomyopathy: clinical relevance and response to pharmacologic therapy. J Am Coll Cardiol. 2010; 55:213-220.

33. Cardinale D, Sandri MT, Colombo A, Colombo N, Boeri M, Lamantia G, Civelli M, Peccatori F, Martinelli G, Fiorentini C, Cipolla CM. Prognostic value of troponin I in cardiac risk stratification of cancer patients undergoing high-dose chemotherapy. Circulation. 2004; 109:27492754.

34. Bloom MW, Hamo CE, Cardinale D, Ky B, Nohria A, Baer L, Skopicki H, Lenihan DJ, Gheorghiade M, Lyon AR, Butler J. Cancer Therapy-Related Cardiac Dysfunction and Heart Failure: Part 1: Definitions, Pathophysiology, Risk Factors, and Imaging. Circ Heart Fail. 2016; 9:e02661.

35. Armenian SH, Lacchetti C, Barac A, et al. Prevention and Monitoring of Cardiac Dysfunction in Survivors of Adult Cancers: American Society of Clinical Oncology Clinical Practice Guideline. J Clin Oncol. 2017; 35:893-911.

36. Jacob S, Pathak A, Franck D, et al. Early detection and prediction of cardiotoxicity after radiation therapy for breast cancer: the BACCARAT prospective cohort study. Radiat Oncol. 2016; 11:54.

37. Satoh K, Takahashi J, Matsumoto Y, Tatebe S, Aoki T, Kikuchi Y, Hao K, Ohyama K, Nogi M, Suda A, Kasahara S, Sato K, Ichijo S, Shimokawa H. European Society of Cardiology (ESC) Annual Congress Report From Barcelona 2017. Circ J. 2017; 81:1758-1763.

38. Caletti S, Paini A, Coschignano MA, De Ciuceis C, Nardin M, Zulli R, Muiesan ML, Salvetti M, Rizzoni D. Management of VEGF-Targeted Therapy-Induced Hypertension. Curr Hypertens Rep. 2018; 20:68.

39. Williams B, Mancia G, Spiering W, et al. 2018 ESC/ESH Guidelines for the management of arterial hypertension. Eur Heart J. 2018; 39:3021-3104.

40. Das D, Asher A, Ghosh AK. Cancer and Coronary Artery Disease: Common Associations, Diagnosis and Management Challenges. Curr Treat Options Oncol. 2019; 20:46.

41. Aghel N, Delgado DH, Lipton JH. Cardiovascular toxicities of BCR-ABL tyrosine kinase inhibitors in chronic myeloid leukemia: preventive strategies and cardiovascular surveillance. Vasc Health Risk Manag. 2017; 13:293-303.

42. Darby SC, Ewertz M, McGale P, et al. Risk of ischemic heart disease in women after radiotherapy for breast cancer. N Engl J Med. 2013; 368:987-998.

43. Plana JC, Galderisi M, Barac A, et al. Expert consensus for multimodality imaging evaluation of adult patients during and after cancer therapy: a report from the American Society of Echocardiography and the European Association of Cardiovascular Imaging. J Am Soc Echocardiogr. 2014; 27:911-939.

44. Soultati A, Mountzios G, Avgerinou C, Papaxoinis G, Pectasides D, Dimopoulos MA, Papadimitriou C. Endothelial vascular toxicity from chemotherapeutic agents: preclinical evidence and clinical implications. Cancer Treat Rev. 2012; 38:473-483.

45. Valgimigli M, Bueno H, Byrne RA, et al. 2017 ESC focused update on dual antiplatelet therapy in coronary artery disease developed in collaboration with EACTS: The Task Force for dual antiplatelet therapy in coronary artery disease of the European Society of Cardiology (ESC) and of the European Association for Cardio-Thoracic Surgery (EACTS). Eur Heart J. 2018; 39:213-260.

46. Kravchenko J, Berry M, Arbeev K, Lyerly HK, Yashin A, Akushevich I. Cardiovascular comorbidities and survival of lung cancer patients: Medicare data based analysis. Lung Cancer. 2015; 88:85-93.

47. Romitan DM, Radulescu D, Berindan-Neagoe I, Stoicescu L, Grosu A, Radulescu L, Gulei D, Ciuleanu TE. Cardiomyopathies and Arrhythmias Induced by Cancer Therapies. Biomedicines. 2020; 8.

48. Al-Khatib SM, Stevenson WG, Ackerman MJ, et al. 2017 AHA/ACC/HRS Guideline for Management of Patients With Ventricular Arrhythmias and the Prevention of Sudden Cardiac Death: A Report of the American College of Cardiology/American Heart Association Task Force on Clinical Practice Guidelines and the Heart Rhythm Society. J Am Coll Cardiol. 2018; 72:e91-e220.

49. Jamieson SW, Kapelanski DP, Sakakibara N, Manecke GR, Thistlethwaite PA, Kerr KM, Channick RN, Fedullo PF, Auger WR. Pulmonary endarterectomy: experience and lessons learned in 1,500 cases. The Annals of Thoracic Surgery. 2003; 76:1457-1464.

50. Galie N, Humbert M, Vachiery JL, et al. 2015 ESC/ERS Guidelines for the diagnosis and treatment of pulmonary hypertension: The Joint Task Force for the Diagnosis and Treatment of Pulmonary Hypertension of the European Society of Cardiology (ESC) and the European Respiratory Society (ERS): Endorsed by: Association for European Paediatric and Congenital Cardiology (AEPC), International Society for Heart and Lung Transplantation (ISHLT). Eur Heart J. 2016; 37:67-119.

51. Agnelli G, Becattini C, Bauersachs R, et al. Apixaban versus Dalteparin for the Treatment of Acute Venous Thromboembolism in Patients with Cancer: The Caravaggio Study. Thromb Haemost. 2018; 118:16681678.

Received July 8, 2021; Revised July 19, 2021; Accepted July 21, 2021.

${ }^{\S}$ These authors contributed equally to this work.

*Address correspondence to:

Yaosheng Wang, Department of Cardiology, Xinhua Hospital Affiliated to Shanghai Jiao Tong University School of Medicine, Shanghai, China.

E-mail: wangyaosheng@xinhuamed.com.cn

Released online in J-STAGE as advance publication July 23, 2021. 